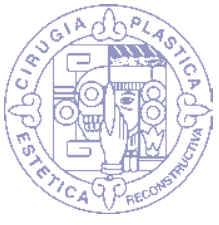

TRABAJO DE INVESTIGACIÓN

doi: $10.35366 / 98338$

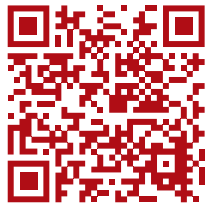

Palabras clave: Colgajo, factores, fracaso.

Keywords: Flap, factors, failure.

${ }^{*}$ Médico residente de cirugía general.

‡ Cirujano plástico y reconstructivo, jefatura de cirugía plástica y reconstructiva. $\S$ Cirujano oncólogo, profesor titular de postgrado de cirugía general.

Centenario Hospital Miguel Hidalgo.

Recibido:

27 agosto 2020

Aceptado:

14 septiembre 2020

\section{Factores pronósticos asociados a fracaso de colgajos}

\author{
Prognostic factors associated to flap failure
}

Dr. Erick Zúñiga-Garza,* Dr. Sergio de la Cruz-Reyes, ${ }^{\star}$ Dr. Efrén Flores-Álvarez ${ }^{\S}$

\begin{abstract}
RESUMEN
Las indicaciones para realizar un colgajo son variadas, en su estudio siempre se enfatiza la técnica; sin embargo, existe poca bibliografía relacionada con los factores asociados al fracaso de un colgajo. El objetivo de este trabajo fue identificar los factores asociados al fracaso de un colgajo en pacientes operados en el Centenario Hospital «Miguel Hidalgo». Revisamos los expedientes clínicos del hospital para identificar a los casos de más de 17 años sometidos a cirugía, y que se les realizó algún colgajo en el lapso de junio de 2013 a julio de 2019. Incluyó un total de 124 pacientes: 69 mujeres (55.6\%) y 55 hombres (44.4\%), con una edad promedio de $56.5 \pm 19.7$ años. La viabilidad del colgajo se demostró en 117 pacientes (94.4\%) y siete colgajos (5.6\%) no fueron viables, 29 presentaron alguna complicación, la más común fue la dehiscencia. Concluimos que en nuestro estudio los factores asociados al fracaso de un colgajo son la linfopenia $(p=0.04)($ OR 3.060) y el tabaquismo $(\mathrm{p}=0.03)(\mathrm{OR} 2.460)$.
\end{abstract}

\section{ABSTRACT}

The indications for making a flap are varied, when analyzed the technique is always emphasized. However, there is little literature related to factors associated with flap failure. The objective of this work was to identify the factors associated with flap failure in patients operated in the Miguel Hidalgo Centennial Hospital. We reviewed the clinical records of the hospital to identify patients over 17 years of age who underwent surgery and had a flap made in the period from June 2013 to July 2019. It included a total of 124 patients: 69 women (55.6\%) and 55 men (44.4\%), with an age average of $56.5 \pm 19.7$ years. Flap viability was demonstrated in 117 patients (94.4\%) and 7 flaps (5.6\%) were non-viable. Twenty-nine patients presented complications, dehiscence being the most common. We conclude that in our study the factors associated with flap failure are lymphopenia $(p=0.04)(O R 3.060)$ and smoking $(p=0.03)($ OR 2.460).

\section{INTRODUCCIÓN}

L a piel es el órgano de mayor extensión del $\_$cuerpo, posee una rica red de arterias y venas subcutáneas; esta abundancia de vasos sanguíneos permite la confección de diversos colgajos. Un colgajo se define como una unidad de tejido que es transferido desde el sitio donante al sitio receptor con su respectiva irrigación. ${ }^{1}$ Existen múltiples indicaciones para la realización de un colgajo, ${ }^{2}$ entre las más comunes están la enfermedad maligna y los traumatismos. ${ }^{1}$ En el estudio de estos se prioriza en la técnica quirúrgica y en esta técnica reside el éxito de los colgajos. ${ }^{3}$ Se ha descrito que diversos factores se asocian a las complicaciones de una cirugía, que incluyen: desnutrición, insuficiencia renal crónica, diabetes mellitus y exposición a nicotina, entre otros. ${ }^{4-6}$

El objetivo de nuestro estudio fue identificar los factores que se asocian al fracaso de un colgajo en pacientes operados en el Centenario Hospital «Miguel Hidalgo».

\section{MATERIAL Y MÉTODO}

Se trata de un estudio observacional retrospectivo en el que se realizó análisis estadístico con el programa SPSS versión 25 (SPSS Inc., Chicago, IL), así como una búsqueda en el
Citar como: Zúñiga-Garza E, de la Cruz-Reyes S, Flores-Álvarez E. Factores pronósticos asociados a fracaso de colgajos. Cir Plast. 2020; 30 (3): 144-148. https://dx.doi.org/10.35366/98338 
expediente clínico electrónico del Centenario Hospital «Miguel Hidalgo» para identificar casos sometidos a cirugía en los que se realizó algún colgajo en el periodo de junio de 2013 a julio de 2019; en total, 217 expedientes. Se incluyeron todos los pacientes de 17 años y más a los que se les realizó un colgajo. Se excluyeron expedientes incompletos, con pérdida de seguimiento y los colgajos realizados por un servicio ajeno al de cirugía; en total se excluyeron 93 pacientes.

Las variables analizadas fueron edad, tiempo de cirugía, sangrado, hemoglobina, leucocitos y linfocitos. Las variables cualitativas fueron sexo, enfermedad crónico-degenerativa, tabaquismo, uso de esteroides, etiología y uso de antibióticos y se investigó la asociación con complicaciones o fracaso del colgajo.

Para analizar los resultados se utilizó la estadística descriptiva. Las variables cuantitativas se dicotomizaron y se analizó la relación con el fracaso de un colgajo usando la prueba estadística $\chi^{2}$. Asimismo, se analizó la relación de las variables cualitativas usando la prueba estadística $\chi^{2}$. Un valor de $p<0.05$ denotó significancia estadística.

\section{RESULTADOS}

Se incluyeron 124 casos en los que se realizó un colgajo: 69 mujeres (55.6\%) y 55 hombres (44.4\%), con una edad promedio de $56.5 \pm 19.7$ años.

Se estudiaron las enfermedades crónicodegenerativas presentes y se encontró que 54 pacientes $(43.5 \%)$ tenían algún tipo de comorbilidad, la más común fue diabetes meIlitus (23.4\%), seguida de hipertensión arterial sistémica (9.7\%), cáncer (4\%), demencia (0.9\%) y enfermedad renal crónica (0.9\%). El tabaquismo se observó en 48 pacientes (38.7\%) y el uso crónico de esteroides en dos (1.6\%) (Tabla 1).

La localización más frecuente fue la cara en 76 pacientes $(61.3 \%)$, seguida de los miembros inferiores en 19 (15.3\%), mama en $15(12.1 \%)$, abdomen en tres $(2.4 \%)$ y en otra en seis $(4 \%)$.

La etiología más común fue la maligna en 79 pacientes $(63.7 \%)$, origen traumático en 23 (18.5\%), infeccioso en 11 (8.9\%) y por otro tipo de enfermedad benigna en 11 (8.9\%).

Se analizó la etiología maligna, encontrando que el tumor más común fue el carcinoma basocelular en 40 pacientes (32.3\%), seguido del carcinoma epidermoide en 18 (14.5\%), cáncer de mama en 12 (9.7\%), melanoma en seis $(4.8 \%)$ y otros en tres $(2.4 \%)$.

En cuanto al tiempo quirúrgico, en 95 sujetos $(76.6 \%)$ el procedimiento quirúrgico duró menos de 120 minutos y en 29 (23.4\%) más de 120 minutos (Tabla 2).

Un total de 29 casos (23\%) desarrollaron algún tipo de complicación, la más común fue la dehiscencia en nueve (7.3\%), necrosis en ocho $(6.5 \%)$, costra hemática en cinco $(4 \%)$, epidermólisis en tres (2.4\%), hematoma en dos (1.6\%) e infección del sitio quirúrgico en dos (1.6\%). En cuanto al sangrado operatorio, en

\begin{tabular}{lc}
\multicolumn{2}{|c}{ Tabla 1: Características generales } \\
de los pacientes. N = 124.
\end{tabular}




\begin{tabular}{|c|c|}
\hline & n (\%) \\
\hline \multicolumn{2}{|l|}{ Localización anatómica } \\
\hline • Cara & $76(61.3)$ \\
\hline - Miembros pélvicos & $19(15.3)$ \\
\hline - Miembro torácico & $5(4.1)$ \\
\hline • Mama & $15(12.1)$ \\
\hline - Abdomen & $3(2.4)$ \\
\hline - Otra & $6(4.0)$ \\
\hline \multicolumn{2}{|l|}{ Etiología del defecto } \\
\hline • Maligna & 79 (63.7) \\
\hline - Benigna & $11(8.9)$ \\
\hline - Traumático & $23(18.5)$ \\
\hline - Infeccioso & $11(8.9)$ \\
\hline Reporte de patología & $(\mathrm{n}=79,100 \%)$ \\
\hline - Carcinoma basocelular & $40(50.6)$ \\
\hline - Carcinoma epidermoide & $18(22.8)$ \\
\hline - Carcinoma ductal & $12(13.5)$ \\
\hline •Melanoma & $6(7.5)$ \\
\hline \multicolumn{2}{|l|}{ Tiempo quirúrgico } \\
\hline$\cdot \leq 120$ minutos & $95(76.6)$ \\
\hline - $>120$ minutos & $29(23.4)$ \\
\hline \multicolumn{2}{|l|}{ Sangrado (mL) } \\
\hline$\cdot>100$ & 14 (11.3) \\
\hline$\cdot \leq 100$ & $110(88.7)$ \\
\hline \multicolumn{2}{|l|}{ Antibiótico profiláctico } \\
\hline • Sí & $30(24.2)$ \\
\hline • No & $94(75.8)$ \\
\hline \multicolumn{2}{|l|}{ Antibiótico } \\
\hline - Cefalotina & $27(87.1)$ \\
\hline - Ceftriaxona & $3(9.7)$ \\
\hline - Clindamicina & $1(3.2)$ \\
\hline
\end{tabular}

110 pacientes (88.7\%) fue menos de $100 \mathrm{~mL}$ y en 14 (11.3\%) fue mayor de $100 \mathrm{~mL}$ (Tabla 3).

A 30 individuos (23.4\%) se les aplicó profilaxis antibiótica y a 94 (75.8\%) no se les aplicó. El antibiótico más utilizado fue la cefalotina en $27(87.1 \%)$, seguido de ceftriaxona en dos $(9.7 \%)$ y clindamicina en uno $(0.8 \%)$ (Tabla 2$)$.

Entre las variables de laboratorio preoperatorio estudiadas, se encontró que 40 pacientes $(32.3 \%)$ tuvieron una hemoglobina $\leq 12.9 \mathrm{~g} /$ $\mathrm{dL}$ y en $84(67.7 \%)$ de $>12.9 \mathrm{~g} / \mathrm{dL}$. En tres casos $(2.4 \%)$ se tuvo un conteo leucocitario absoluto $\leq$ de $3.500010^{3} / \mu \mathrm{L}$, en tanto que en $121(97.6 \%)$ fue de $>3.500010^{3} / \mu$ L. En 31 pacientes (25\%) hubo un conteo linfocitario $\leq$ $1.60010^{3} / \mu \mathrm{L}$ mientras que en 93 (95\%) fue > $1.60010^{3} / \mu \mathrm{L}$.

El objetivo final fue la supervivencia del colgajo. De los 124 que se realizaron, 117 (94.4\%) sobrevivieron y siete (5.6\%) no fueron viables.

Después de la aplicación de la prueba $\chi^{2}$, se encontró que las variables que tuvieron asociación con significancia estadística para la no viabilidad de los colgajos fueron: tabaquismo $(p=0.03)(O R 5.460)$ y linfopenia $(p=0.04)$ (OR 3.960). La diabetes mellitus $(p=0.06)$, a pesar de no alcanzar una significancia estadística, tuvo una tendencia clínica asociada al fracaso de un colgajo.

\section{DISCUSIÓN}

La historia de los colgajos inició desde tiempos inmemorables, la descripción del primer colgajo se le atribuye a Sushruta Samhita en el año 600 a.C. ${ }^{7}$ Debido a la definición del término angiosoma, su distribución y aplicaciones clínicas, se han podido diseñar los colgajos actuales, ${ }^{8}$ los cuales cada vez son más complejos y exigentes desde el punto de vista técnico.

Los colgajos pueden clasificarse según su composición, vascularización, método de transferencia y diseño. ${ }^{9}$ En la fisiopatología del fracaso de un colgajo, se han identificado factores extrínsecos (infección, arterioesclerosis, hipotensión, desnutrición) y factores intrínsecos (compresión, tensión, trombosis de la anastomosis o torsión del pedículo. ${ }^{10}$

En nuestro estudio, se buscaron de manera intencional factores asociados al fracaso de un colgajo para identificarlos, poder corregirlos a tiempo y así lograr una mayor tasa de colgajos viables.

Se fijó un corte en el nivel de hemoglobina de $12.9 \mathrm{~g} / \mathrm{dL}$, según la definición de anemia. ${ }^{11}$ Se fijó un corte en el nivel de leucocitos absolutos de $3.50010^{3} / \mu \mathrm{L}^{12}$ se fijó un corte en el nivel de linfocitos absolutos de $1.60010^{3}$ / $\mu \mathrm{L} .{ }^{12}$ De manera arbitraria se tomó un corte en el tiempo de cirugía (120 minutos) y en el volumen de sangrado $(200 \mathrm{~mL}$ ).

En nuestros resultados se reportó una viabilidad de los colgajos del 94.4\%. Harashina ${ }^{3}$ 
reportó una viabilidad del $94.8 \%$, Brunetti ${ }^{13}$ del 93.1\%, Bozikov y Arnez ${ }^{14}$ del $94.3 \%$, Crawley ${ }^{15}$ del $95.2 \%$, por lo que consideramos que nuestra tasa de éxito concuerda con lo reportado en la bibliografía internacional.

En nuestra serie, la indicación más común para la realización de un colgajo fue la malignidad y la localización más común fue la cara, esto concuerda con la bibliografía consultada. ${ }^{13,15}$

Sólo en $23.4 \%$ de los pacientes se cumplieron de manera completa las indicaciones de los centros para el control y la prevención de enfermedades. ${ }^{16} \mathrm{~A}$ pesar de esto, este factor no tuvo asociación con complicaciones ni fracaso de los colgajos; sin embargo, estas cifras son alarmantes.

A pesar de que en otras referencias se hace énfasis en la técnica quirúrgica, en nuestro trabajo se estudió una población, diseño, indicaciones y etiología heterogéneos, con la finalidad de encontrar un factor común asociado al fracaso de los colgajos y así poder actuar para modificarlo previo a la realización de un colgajo.

\section{Limitaciones}

En primera instancia, se trata de un estudio retrospectivo. Por la naturaleza del tipo de estudio, sólo nos basamos en la información contenida en el expediente clínico, lo que limita el seguimiento adecuado de los casos.

\begin{tabular}{|c|c|}
\hline \multicolumn{2}{|c|}{$\begin{array}{l}\text { Tabla 3: Complicaciones de } \\
\text { los colgajos. } N=124 \text {. }\end{array}$} \\
\hline & $\mathrm{n}(\%)$ \\
\hline \multicolumn{2}{|l|}{ Complicación } \\
\hline • No & $95(80.5)$ \\
\hline - Sí & 29 (19.5) \\
\hline Tipo de complicaciones & $\mathrm{n}=29,100 \%$ \\
\hline - Dehiscencia & $9(31.0)$ \\
\hline - Necrosis & $8(27.6)$ \\
\hline - Epidermólisis & $3(10.3)$ \\
\hline • Hematoma & $2(6.9)$ \\
\hline - Costra hemática & $5(17.2)$ \\
\hline - Infección & $2(6.8)$ \\
\hline
\end{tabular}

Sería interesante poder intervenir en los factores identificados asociados al fracaso de un colgajo y poder prevenir dicho fracaso.

\section{CONCLUSIÓN}

En una población mexicana se observó que el tabaquismo $(p=0.03)(O R 5.460)$ y la linfopenia $(p=0.04)$ (OR 3.960) son factores de riesgo asociados a fracaso de un colgajo.

\section{REFERENCIAS}

1. Thorne $\mathrm{CH}$, Chung KC, Gosain AK, Gurtner GC, Mehrara BJ, Rubin JP et al. Grabb and Smith's plastic surgery, Seventh edition. Wolters Kluwer Health Adis, 2013.

2. Tschoi M, Hoy EA, Granick MS. Skin flaps. Clin Plast Surg 2005; 32 (2):261-273.

3. Harashina T. Analysis of 200 free flaps. Br J Plast Surg 1988; 41 (1): 33-36.

4. Arita-Zelaya AC, Fierro-Arias L, Arellano-Mendoza I, Ponce-Olivera RM, Peniche Castellanos A. Frecuencia de factores de riesgo y etiología de infecciones postquirúrgicas en cirugía dermatológica, Hospital General de México. Rev Med Hosp Gen Mex 2009; 72 (3): 136-141.

5. Nolan J, Jenkins RA, Kurihara K, Schultz RC. The acute effects of cigarette smoke exposure on experimental skin flaps. Plast Reconstr Surg 1985; 75 (4): 544-551.

6. Chang LD, Buncke G, Slezak S, Buncke HJ. Cigarette smoking, plastic surgery, and microsurgery. J Reconstr Microsurg 1996; 12 (07): 467-474.

7. Sushruta S. An english translation of the Sushruta Samhita based on original Sanskrit text. 1963.

8. Taylor GI, Palmer JH. The vascular territories (angiosomes) of the body: experimental study and clinical applications. J Plast Reconstr Aesthet Surg 1987; 40 (2): 113-141.

9. Patel KG, Sykes JM. Concepts in local flap design and classification. Op Tech Otolaryngol Head Neck Surg 2011; 22 (1): 13-23.

10. Kerrigan CL. Skin flap failure: pathophysiology. Plast Reconstr Surg 1983; 72 (6): 766-777.

11. World Health Organization. Hemoglobin concentrations for the diagnosis of anemia and assessment of severity. Vitamin and Mineral Nutrition Information System. Geneva, World Health Organization, 2011.

12. Young GA, Hill GL. Assessment of protein-calorie malnutrition in surgical patients from plasma proteins and anthropometric measurements. Am J Clin Nut 1978; 31 (3): 429-435.

13. Brunetti B, Barone M, Tenna S, Salzillo R, Segreto F, Persichetti P. Pedicled perforator-based flaps: Risk factor analysis, outcomes evaluation and decisional algorithm based on 130 consecutive reconstructions. Microsurgery 2020; 40 (5): 545-552.

14. Bozikov K, Arnez ZM. Factors predicting free flap complications in head and neck reconstruction. J Plast Reconstr Aesthet Surg 2006; 59 (7): 737-742. 
15. Crawley MB, Sweeny L, Ravipati P, Heffelfinger R, Krein $\mathrm{H}$, Luginbuhl A et al. Factors associated with free flap failures in head and neck reconstruction. Otolaryngol Head Neck Surg 2019; 161 (4): 598-604.

16. Mangram AJ, Horan TC, Pearson ML, Silver LC, Jarvis WR. Guideline for Prevention of Surgical Site Infection, 1999. Centers for Disease Control and Prevention (CDC) Hospital Infection Control Practices Advisory Committee. Am J Infect Control 1999; 27 (2): 97-132.
Correspondencia:

\section{Dr. Erick Zúñiga Garza}

Calle Consuelo R Macías 402 interior A,

Col. José Luis Macías, Rincón de Romos, 20400, Aguascalientes

E-mail: erickzunigagarza5@gmail.com

Conflicto de intereses: Los autores declaran no tener conflicto de intereses. 